\title{
Progression de l'ambroisie et risques sanitaires: la Suisse résistera-t-elle à cette invasion?
}

\author{
P. Taramarcaz ${ }^{a}$, C. Lambelet ${ }^{b, e}$, B. Clot $^{c, e}$, C. Keimerd, e , C. Hausera, e
}

a Service d'Allergologie et Immunologie, Hôpital Universitaire de Genève

b Conservatoire et Jardin botaniques de la Ville de Genève

c MétéoSuisse, Biométéorologie et environnement, Station aérologique, Payerne

d Service de l'agriculture, Station phytosanitaire, Genève

e Group Ambroisie de Genève

Correspondance:

Prof. Dr Conrad Hauser

Service d'Allergologie et Immunologie

Hôpital Universitaire de Genève

24, rue Micheli-du-Crest

CH-1211 Genève 14

E-mail:

Conrad.Hauser@medecine.unige.ch
Le but de ce papier est d'alerter les médecins suisses sur les risques sanitaires et environnementaux lié à l'ambroisie (Ambrosia artemisiifolia). Il a été rédigé par des experts en botanique, en aérobiologie, en agronomie et en allergologie.

Les zones européennes fortement infestées par l'ambroisie sont certaines parties de l'Autriche, la Hongrie, la Bulgarie, certains états issus de la fragmentation de la Yougoslavie et, plus près de chez nous, la région lyonnaise (France) et la vallée du Pô (Italie). Dans les zones fortement colonisée par cette mauvaise herbe, près de $12 \%$ de la population souffre d'allergie aux pollens d'ambroisie (rhinite et asthme). La Suisse commence également à être envahie par cette plante herbacée. Les capteurs de pollens implantés sur de multiples sites du territoire helvétique enregistrent la quantité de pollens d'ambroisie depuis quelques années. Ces taux restent toutefois encore bas dans notre pays, mais peuvent atteindre localement des seuils critiques qui provoquent des symptômes chez les individus allergiques. L'allergie à l'ambroisie reste cependant encore rare en Suisse. Devant de l'augmentation des taux de pollen d'ambroisie durant plusieurs années consécutives, la recherche systématique de foyer locaux d'ambroisie a été initiée à Genève et au Tessin. Mis à part quelques foyers mineurs répertoriés sur l'ensemble du territoire helvétique, les plus grandes accumulations d'ambroisie sont localisées à Genève, sur la côte ouest du Lac Léman (canton de Vaud) et au sud du canton du Tessin. L'envahissement de l'ambroisie progresse préférentiellement le long des axes autoroutiers et des voies de chemin de fer. Le transport de terre contaminée et la location de machines agricoles provenant de régions infestées contribuent également à la propagation de cette plante envahissante. Certains lots de semences et la nourriture pour oiseaux peuvent aussi être contaminés et être responsable de foyer locaux d'ambroisie. Les services phytosanitaires genevois diffusent depuis quelques années l'information permettant d'identifier l'ambroisie et coordonnent les mesures d'éradication de cette plante néophyte envahissante. De nouvelles méthodes d'éradication sont étudiées à différents niveaux. Il est urgent de pouvoir implémenter ces méthodes durant cette phase précoce de colonisation. L'expérience d'autres pays limitrophes, déjà envahis par cette herbacée, nous a enseigné que l'éradication complète de l'ambroisie est virtuellement impossible, une fois qu'une zone est fortement colonisée. Les autorités fédérales doivent s'engager à coordonner la dissémination de l'information concernant le risque sanitaire et agricole lié à l'envahissement de cette mauvaise herbe et prévenir sa propagation en proposant des mesures adéquates. Une future législation fédérale pourrait offrir un cadre adéquat pour une prévention efficace, en interdisant par exemple l'importation des graines pour oiseaux et les lots de semences contaminées par des graines d'ambroisie. Les coûts engendrés par des mesures de prévention, à ce stade précoce de l'envahissement de l'ambroisie, sont largement inférieurs à ceux engendrés par le traitement d'une fraction significative de la population suisse ayant développé une rhinite et un asthme allergique aux pollens d'ambroisie (par exemple les coûts estimés pour traiter 100000 patients allergiques atteignent 100 millions de CHF par année). Les zones faiblement colonisées peuvent, en l'espace de seulement quelques années, se transformer en zones fortement colonisées. De ce fait il ne faut pas rater l'opportunité actuelle de prévenir une plus forte colonisation du territoire helvétique par l'ambroisie.

Une revue plus détaillée sur ce sujet du même group d'auteurs apparaîtra bientôt dans le Swiss Medical Weekly. L'accès à la version électronique est gratuit (www.smw.ch). 